\title{
Glyco-engineering strategies for the development of therapeutic enzymes with improved efficacy for the treatment of lysosomal storage diseases
}

\author{
Doo-Byoung Oh ${ }^{1,2, *}$ \\ ${ }^{1}$ Synthetic Biology and Bioengineering Research Center, Korea Research Institute of Bioscience \& Biotechnology (KRIBB), ${ }^{2}$ Biosystems and \\ Bioengineering Program, University of Science and Technology (UST), Daejeon 34141, Korea
}

\begin{abstract}
Lysosomal storage diseases (LSDs) are a group of inherent diseases characterized by massive accumulation of undigested compounds in lysosomes, which is caused by genetic defects resulting in the deficiency of a lysosomal hydrolase. Currently, enzyme replacement therapy has been successfully used for treatment of 7 LSDs with 10 approved therapeutic enzymes whereas new approaches such as pharmacological chaperones and gene therapy still await evaluation in clinical trials. While therapeutic enzymes for Gaucher disease have $\mathrm{N}$-glycans with terminal mannose residues for targeting to macrophages, the others require $\mathrm{N}$-glycans containing mannose-6-phosphates that are recognized by mannose-6-phosphate receptors on the plasma membrane for cellular uptake and targeting to lysosomes. Due to the fact that efficient lysosomal delivery of therapeutic enzymes is essential for the clearance of accumulated compounds, the suitable glycan structure and its high content are key factors for efficient therapeutic efficacy. Therefore, glycan remodeling strategies to improve lysosomal targeting and tissue distribution have been highlighted. This review describes the glycan structures that are important for lysosomal targeting and provides information on recent glyco-engineering technologies for the development of therapeutic enzymes with improved efficacy. [BMB Reports 2015; 48(8): 438-444]
\end{abstract}

\section{INTRODUCTION}

A lysosome is an acidic cellular organelle containing more than 60 hydrolytic enzymes for digestion and recycling of vari-

${ }^{*}$ Corresponding author. Tel: +82-42-860-4457; Fax: +82-42-8798494; E-mail: dboh@kribb.re.kr

http://dx.doi.org/10.5483/BMBRep.2015.48.8.101

Received 21 May 2015

Keywords: Enzyme replacement therapy, Glcyan, Glyco-engineering, Lysosomal storage disease, Mannose-6-phosphate, Therapeutic enzymes ous macromolecules. Lysosomal storage diseases (LSDs) are caused by genetic defects resulting in deficiencies of these enzymes, which leads to massive accumulation of undigested macromolecules in lysosomes (1). This accumulation induces lysosomal and cellular dysfunctions progressively leading to the failures of multiple tissues and organs including the brain, viscera, bone and connective tissues. The LSD patients seem normal at birth but develop clinical manifestations (such as abnormal enlargement of organs, coarsening of hair and facial features, and skeletal and central nervous system defects) mainly in infancy/childhood and often end up with early death (1-4).

For treatment of LSDs, bone marrow transplantation, enzyme replacement therapy (ERT), and substrate reduction technology are currently available $(1,2,4)$. Although bone marrow transplantation was the only therapy available in the past, it is now mainly used in the cases where ERT does not work $(1,2)$. Substrate reduction therapy uses an inhibitor for an enzyme involved in the synthesis of the accumulated compound and has only one approved drug (Miglustat) for Gaucher disease (2). Several novel approaches including pharmacological chaperones and gene therapy are under the development, however these still await evaluation in clinical trials $(2,4)$. In contrast, ERT has 10 approved therapeutic enzymes for 7 LSDs at present after their efficacy and safety have already been confirmed by clinical trials (1-4) (Table 1). However, there still exist several limitations such as immune reactions, low efficiency of lysosomal targeting and difficult delivery to central nervous system. To overcome these limitations, several strategies have been employed to develop next generation therapeutic enzymes, especially focusing on increasing the targeting efficiency and/or crossing the blood brain barrier. Many of these approaches include glycan remodeling in order to change the tissue distribution and improve the cellular uptake and lysosomal targeting. Here, we provides the insights into the glycan structures that are important for lysosomal targeting and current glyco-engineering strategies to improve ERT efficiency.

Mannose-6-phosphate (M-6-P) glycan modification for trafficking to lysosome

A pre-made $\mathrm{N}$-glycan precursor $\left(\mathrm{Gl}_{3} \mathrm{Man}_{9} \mathrm{GlCNac}_{2}\right)$ is attached

ISSN: 1976-670X (electronic edition)

Copyright (C) 2015 by the The Korean Society for Biochemistry and Molecular Biology

(c) This is an open-access article distributed under the terms of the Creative Commons Attribution Non-Commercial License (http://creativecommons.org/licenses/by-nc/4.0) which permits unrestricted non-commercial use, distribution, and reproduction in any medium, provided the original work is properly cited. 
Table 1. Characteristics of the approved therapeutic enzymes for treatment of LSDs

\begin{tabular}{|c|c|c|c|c|c|}
\hline $\begin{array}{l}\text { Disease } \\
\text { (Storage) }\end{array}$ & $\begin{array}{c}\text { Enzyme } \\
\text { (Gene name) }\end{array}$ & $\begin{array}{l}\text { Therapeutics name } \\
\text { (Brand name) }\end{array}$ & $\begin{array}{l}\text { Manufacturing } \\
\text { company }\end{array}$ & $\begin{array}{c}\text { Source of } \\
\text { expression cells }\end{array}$ & Glycan features \\
\hline $\begin{array}{l}\text { Gaucher } \\
\text { (glucosylceramide) }\end{array}$ & $\begin{array}{l}\beta \text {-gluco-cerebrosidase } \\
(G B A)\end{array}$ & $\begin{array}{l}\text { Imiglucerase (Cerezyme) } \\
\text { Velaglucerase alfa (VPRIB) } \\
\text { Taliglucerase alfa (Elelyso) }\end{array}$ & $\begin{array}{l}\text { Sanofi (Genzyme) } \\
\text { Shire HGT } \\
\text { Protalix \& Pfizer }\end{array}$ & $\begin{array}{l}\mathrm{CHO} \\
\text { Human } \\
\text { Carrot }\end{array}$ & $\begin{array}{l}\text { Tri-mannosyl core } \\
\text { High-mannose } \\
\text { Plant pauci-mannose }\end{array}$ \\
\hline $\begin{array}{l}\text { Fabry } \\
\text { (Gb3, LysoGb3) }\end{array}$ & $\alpha$-Galactosidase (GLA) & $\begin{array}{l}\text { Agalsidase beta (Fabrazyme) } \\
\text { Agalsidase alfa (Replagal) }\end{array}$ & $\begin{array}{l}\text { Sanofi (Genzyme) } \\
\text { Shire HGT }\end{array}$ & $\begin{array}{l}\mathrm{CHO} \\
\text { Human }\end{array}$ & $\begin{array}{l}\text { M-6-P glycan (2.9)* } \\
\text { M-6-P glycan }(2.1)^{*}\end{array}$ \\
\hline $\begin{array}{l}\text { Pompe } \\
\text { (glycogen) }\end{array}$ & $\alpha$-Glucosidase (GAA) & $\begin{array}{l}\text { Aglucosidase alfa } \\
\text { (Myozyme/Lumizyme) }\end{array}$ & Sanofi (Genzyme) & $\mathrm{CHO}$ & M-6-P glycan $(0.7)^{*}$ \\
\hline $\begin{array}{l}\text { MPS type I } \\
\text { (DS, HS) }\end{array}$ & Iduronidase (IDUA) & Laronidase (Aldurazyme) & Biomarin & $\mathrm{CHO}$ & M-6-P glycan (2.5)* \\
\hline $\begin{array}{l}\text { MPS type II } \\
\text { (DS, HS) }\end{array}$ & $\begin{array}{l}\text { Iduronate sulfatase } \\
\text { (IDS) }\end{array}$ & Idursulfase (Elaprase) & Shire HGT & Human & M-6-P glycan (3.2)* \\
\hline $\begin{array}{l}\text { MPS type IVA } \\
\text { (KS, C6S) }\end{array}$ & $\begin{array}{l}\text { GalNAc-6-sulfatase } \\
\text { (GALNS) }\end{array}$ & Elosulfase alfa (Vimizim) & Biomarin & $\mathrm{CHO}$ & M-6-P glycan \\
\hline $\begin{array}{l}\text { MPS type VI } \\
\text { (DS, C4S) }\end{array}$ & $\begin{array}{l}\text { GalNAc-4-sulfatase } \\
(A R S B)\end{array}$ & Galsulfase (Naglazyme) & Biomarin & $\mathrm{CHO}$ & M-6-P glycan \\
\hline
\end{tabular}

*The values of M-6-P content (mol/mol enzyme) were obtained from a comparative study (16). Gb3: Globotriaosylceramide, MPS: Mucopolysaccharidosis, DS: Dematan sulfate, HS: Heparan sulfate, KS: Keratan sulfate, C6S: Chondrotin 6-sulfate, C4S: Chondrotin 4-sulfate.

to glycoproteins including lysosomal enzymes by oligosaccharyl transferase in the endoplasmic reticulum (ER) during the co-translational secretion process. After trimming of the three terminal glucoses and one mannose residues of the $\mathrm{N}$-glycan in the ER, the enzymes move to the Golgi apparatus and undergo further glycan trimming and modification. Here, some high-mannose type glycans of lysosomal enzymes are modified to contain M-6-Ps in a two-step reaction (Fig. 1) (5-7). As a first step in the cis-Golgi complex, UDP-GlcNAc:lysosomal enzyme-N-acetylglucosamine-1-phosphotransferase (GlcNAc-1phosphotransferase) recognizes lysosomal enzymes and then transfer GlcNAc-1-phosphate from UDP-GlcNAc to the C6 hydroxyl groups of a specific mannose residue (GlcNAc-1-phosphate-6-O-mannose). GlcNAc-1-phosphotransferase, a heterohexameric complex $\left(\alpha_{2} \beta_{2} \gamma_{2}\right)$ encoded by two genes $(8,9)$, has been known to recognize common conformational structures of lysosomal enzymes in which lysine residues are the major determinants $(10,11)$. In the second step, $\mathrm{N}$-acetylglucosamine-1-phosphodiester $\alpha-\mathrm{N}$-acetylglucosaminidase (uncovering enzyme) removes the outer GlcNAc and leaves a phosphate group linked to the mannose residue (phosphate-6-O-mannose) of the N-glycan, which is called an M-6-P glycan (Fig. 1). The uncovering enzyme encoded by the NAGPA gene is a type I membrane protein and exists as a tetramer that cycles between the trans-Golgi network (TGN) and the plasma membrane (7).

The M-6-P glycans of lysosomal enzymes are recognized by M-6-P receptors (MPRs) for lysosomal delivery. There are two MPRs; a cation-dependent (CD)-MPR with a molecular weight $(\mathrm{MW})$ of $46 \mathrm{kDa}$ and a cation-independent (Cl)-MPR with a $\mathrm{MW}$ of $300 \mathrm{kDa}$ (5-7). MPRs are type I transmembrane glycoproteins distributed over the TGN, endosomes, and plasma membrane $(6,7)$. CD-MPR has a luminal domain of 159 amino acids with M-6-P binding ability, while Cl-MPR contains 15 homologous repeating domains of $\sim 150$ amino acids; domains 3 and 9 are high affinity M-6-P binding sites, and domain 11 provides a binding site for insulin-like growth factor II (IGF II) $(6,7)$. As a multi-functional protein, CI-MPR can bind other ligands including IGF II, retinoic acid, and urokinasetype plasminogen activator receptor $(5,6)$. Studies using knockout mice lacking CD- or Cl-MPR have shown that both MPRs are required to deliver all lysosomal enzymes, as three populations exist, CD-MPR-dependent, Cl-MPR-dependent, and those dependent on both receptors $(5,7)$.

In the TGN, the M-6-P glycans of lysosomal enzymes are recognized by MPRs, which are delivered to the early endosome through clathrin-coated vesicles requiring the interaction of MPRs with coat proteins (Fig. 1) (5-7). As the early endosomes mature to late endosomes, accompanied by $\mathrm{pH}$ decreases, lysosomal enzymes are released from MPRs, which avoids the delivery of MPRs to lysosomes where they can be digested (7). Finally, selective transfer of lysosomal enzymes to lysosomes is achieved by the fusion of late endosomes with lysosomes where they begin to digest the macromolecules at acidic $\mathrm{pH} 5$ which is maintained by a membrane ATP-driven $\mathrm{H}^{+}$pump (7).

\section{Most therapeutic enzymes are delivered to lysosomes by Cl-MPR-mediated endocytosis}

Some fractions of lysosomal enzymes, instead of trafficking to lysosomes via M-6-P pathway, escape binding to MPRs in the TGN and exit to extracellular spaces (Fig. 1) (5-7). Such secreted enzymes can be recaptured by the MPRs located at plasma membranes and delivered to lysosomes by receptor- 


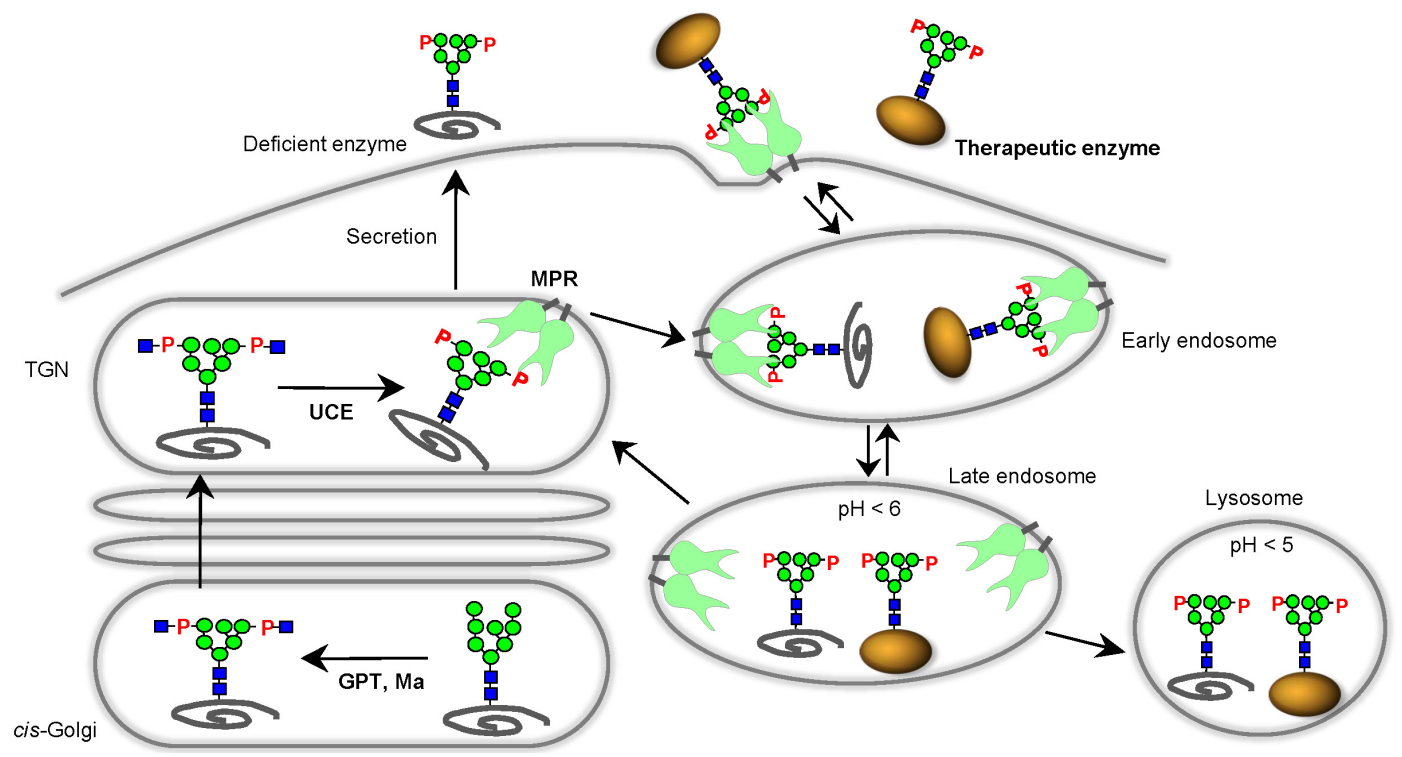

Fig. 1. Trafficking of lysosomal enzymes to lysosomes by the mannose-6-phosphate pathway. Lysosomal enzymes are recognized in the cis-Golgi by GlcNAc-1-phosphotransferase (GPT) transferring a GlcNAc-1-phosphate moiety of UDP-GlcNAc to selected mannoses in high-mannose type glycans of lysosomal enzymes, which is accompanied by mannosidase (Ma) trimming. Subsequently, the outer GlcNAc is removed to leave a phosphate group linked to a mannose residue in the TGN by an uncovering enzyme (UCE), generating M-6-P. Lysosomal enzymes carrying M-6-P glycans are selectively recognized by MPRs in the TGN and then move to early endosomes via clathrin-coated vesicles. At the low $\mathrm{pH}$ of late endosomes, lysosomal enzymes were separated from MPRs and entered the lysosome alone. A portion of lysosomal enzymes escape binding to MPR in the TGN and are secreted outside cells. CI-MPR at the plasma membrane is capable of recapturing such enzymes to lysosomes by an endocytosis mechanism. ERT employs this Cl-MPR-mediated endocytosis pathway to deliver the administrated therapeutic enzymes to lysosomes. Symbols used for glycans are those suggested by the Consortium for Functional Glycomics (http://www.functionalglycomics.org/). Green circle: Mannose, blue square: GlcNAc, P: Phosphate.

mediated endocytosis. Cl-MPR is responsible for this M-6-P-dependent endocytosis in physiological conditions, although small amounts of both CD- and Cl-MPRs exist at plasma membranes. Most of the therapeutic enzymes used in ERT exploit this "secretion-recapture" pathway for cellular uptake and lysosomal targeting. When therapeutic enzymes carrying M-6-P glycans are administrated to the body, they can be recognized and internalized to cells by Cl-MPR at the plasma membranes (7). Seven therapeutic enzymes for the treatment of six LSDs (all enzymes except Imiglucerase, Velaglucerase al$\mathrm{fa}$, and Taliglucerase alfa for Gaucher disease) employ this interaction between their M-6-P glycans and Cl-MPR for targeting to lysosomes (Table 1). They are usually secreted from overexpressing Chinese hamster ovary $(\mathrm{CHO})$ cells or human fibroblasts as glycoproteins containing heterogeneous $\mathrm{N}$-glycans (complex type and M-6-P glycans).

Fabry disease arises from a deficiency in $\alpha$-galactosidase (GLA), which results in accumulation of globotriaosylceramide (Gb3) and lysoGb3. For the treatment, there are two approved GLAs, Agalsidase alfa and Agalsidase beta, which are produced from human fibroblasts and $\mathrm{CHO}$ cells, respectively. They are homodimeric glycoproteins with three $\mathrm{N}$-glycosylation sites in each monomer; one site comprises a complex type glycan with terminal sialic acids, while the other two sites are mainly attached to M-6-P glycans (12). A comparative glycan analysis study showed that Agalsidase beta has higher levels of M-6-P and sialic acid than Agalsidase alfa, which correlates with the better efficacy of Agalsidase beta to reduce the accumulated Gb3 in human Fabry fibroblasts and Fabry ( $\alpha$-galactosidase knockout) mice (12-14). However, significant differences in therapeutic efficacies were not observed in a small clinical study (34 Fabry disease patients) with the treatment of either Agalsidase alfa or Agalsidase beta at the same dose $(0.2$ $\mathrm{mg} / \mathrm{kg}$ ) (15). These results may be related to the marginal difference in M-6-P contents between the two GLAs, which was revealed by a recent comparative study of the M-6-P contents of six therapeutic enzymes (16), with the M-6-P contents of Agalsidase alfa and Agalsidase beta being 2.1 and $2.9 \mathrm{~mol} / \mathrm{mol}$ enzyme, respectively.

Pompe disease, caused by a deficiency in lysosomal acid $\alpha$-glucosidase (GAA), leads to myopathy especially in skeletal muscles, heart, liver, and the nervous system. Early attempts using GAAs prepared from Aspergillus niger and human placenta were not successful due to inappropriate glycan modification and/or low dose $(17,18)$. In contrast, clinical studies using recombinant GAAs produced from the milk of transgenic rabbits and $\mathrm{CHO}$ cell cultures have shown beneficial effects on survival, cardiomyopathy, motor function, and growth $(19,20)$. 
Genzyme researchers carefully compared the in vitro and in vivo properties of these GAAs, and selected the GAA produced from $\mathrm{CHO}$ cells for clinical development (21), leading to the approval of Aglusidase alfa for the treatment of Pompe disease (Table 1).

Mucopolysaccharidoses (MPS) are a group of LSDs that have problems in degradation of mucopolysaccharides (currently called glycosaminoglycans) such as dermatan sulfate, heparan sulfate and chondroitin sulfate (1-3). Currently, four therapeutic enzymes (Laronidase, Idursulfase, Elosulfase alfa, and Galsulfase) are approved for MPS type I, II, IVA, and VI, which are caused by deficiencies in iduronidase, iduronate sulfatase, $\mathrm{N}$-acetylgalactosamine (GalNAc)-6-sulfatase, and GalNAc-4sulfatase, respectively (Table 1) (1-3). Laronidase, Elosulfase al$\mathrm{fa}$, and Galsulfase are produced from $\mathrm{CHO}$ cells while Idursulfase is produced from human fibroblasts. Elosulfase alfa, a recombinant GalNAc-6-sulfatase, was most recently approved in 2014 by the U.S. Food and Drug Administration and the European Medicines Agency for treatment of MPS IVA, which is also known as Morquio A syndrome (3).

\section{Glycan remodeling of $\beta$-glucocerbrosidase led to successful ERT for Gaucher disease}

The first therapeutic enzyme for LSDs was the $\beta$-glucocerebrosidase (Gcase) purified from human placenta for the treatment of Gaucher disease, especially type I without the involvement of the central nervous system (1-3). A deficiency in this enzyme results in accumulation of glycosphingolipids in the macrophages of liver, bone marrow, and spleen. Initial attempts to use placenta-derived Gcase containing complex type glycans, were disappointing due to the fact that the unmodified enzyme was not targeted to the macrophages. After sequential removal of the terminal sialic acid, galactose, and GlcNAc, exposing mannose residues of tri-mannosyl core glycans, it could be successfully delivered to macrophages having mannose receptors (MRs) at the plasma membrane and a MR-mediated endocytosis system. Since then, all of the approved Gcases have been engineered to have $\mathrm{N}$-glycans with terminal mannose residues for targeting to MRs on macrophages $(22,23)$. Because the placenta-derived Gcase suffered from its limited amounts, it was later replaced with recombinant Gcase (Imiglucerase) produced from $\mathrm{CHO}$ cells, and further processed with sequential exoglycosidase digestions for exposing terminal mannose residues. It is the first successful case employing the glyco-engineering strategy to enhance targeting to disease-affected cells $(22,23)$.

Besides Imiglucerase, there are two other approved recombinant Gcases, Velaglucerase alfa (Shire Pharmaceuticals Inc.) and Taliglucerase alfa (Protalix Pharmaceuticals Inc.) (Table 1). Velaglucerase alfa is produced from a human fibroblast carcinoma cell line and manipulated to contain $\mathrm{N}$-glycans with terminal mannose residues by kifunensine treatment to the culture medium. Kifunensine treatment blocks the mannose trimming step by inhibiting mannosidase I, which leads to gen- eration of high-mannose type glycan $\left(\mathrm{Man}_{6-9} \mathrm{GlcNAc}_{2}\right)$ (22). Taliglucerase alfa is produced from plant cells (carrot root cells) and is targeted to plant storage vacuoles, which generates plant pauci-mannose type $\mathrm{N}$-glycans $\left(\mathrm{Man}_{3} \mathrm{Xy}_{1} \mathrm{Fuc}_{1} \mathrm{GlCNAC}_{2}\right)$ having exposed terminal mannose residues without an additional processing step (22). Although there are apparent variations in $\mathrm{N}$-glycan structures, a comparison study of all three enzymes showed similar results in in vitro enzyme activity, ex vivo cellular uptake, and in vivo tissue distribution assays (22). However, in another study using monocytes isolated from a Gaucher patient, Taliglucrease alfa had a lower efficiency of uptake than the others (24). The concern that the prolonged use of Taliglucerase alfa containing the plant-specific glycan structures $(\beta-(1,2)$-xylose and core $\alpha$ - $(1,3)$-fucose) may induce an immune reaction such as an allergy still remains (23). In addition, Velaglucerase alfa has a concern related to its longer mannose chains, which was shown to bind more efficiently to an undesirable target, mannose binding lectin, in serum (25).

Glyco-engineering strategies to increase M-6-P glycan content As glyco-engineered Gcases displayed successful efficacy through the targeting of MRs on macrophages, glyco-engineering strategies to increase the M-6-P glycan content have also been actively applied to the development of therapeutic enzymes with improved lysosomal targeting. Such approaches are especially focused on recombinant GAA for Pompe disease because it has a very low content of M-6-Ps $(0.7 \mathrm{~mol} / \mathrm{mol}$ enzyme) compared with other enzymes $(2.1-3.2 \mathrm{~mol} / \mathrm{mol}$ enzyme) (Table 1); high doses of GAA (20-100 mg/kg) can only partially reduce the glycogen level in skeletal muscles, which are the most severely affected tissues and known to have a low level of Cl-MPR (26). In order to overcome this limitation, Genzyme researchers introduced additional M-6-P moieties onto GAA by enzymatic engineering or chemical conjugation of M-6-P glycans.

In an enzymatic engineering approach (27), the recombinant GAA carrying high-mannose type glycans was purified from the medium of $\mathrm{CHO}$ cells cultured in the presence of kifunesine (a mannosidase I inhibitor). It was further engineered to have additional M-6-P glycans by two-step enzyme reactions comprising the first GlcNAc-1-phosphotransferase reaction (for the generation of a GlcNAc-1-phosphate-6-O-mannose structure), and the second uncovering enzyme reaction (for removal of the outer GlcNAc) in order to generate a M-6-P moiety (phosphate-6-O-mannose). This engineered GAA (referred to as HP-GAA) was shown to have a relatively higher M-6-P content $(\sim 3.5 \mathrm{~mol} / \mathrm{mol}$ enzyme) compared with that $(\sim 1.3 \mathrm{~mol} / \mathrm{mol}$ enzyme) of the unmodified GAA. HP-GAA showed the increased binding to MPR and subsequent enhanced uptake by cells in culture (21). However, in vivo experiments using Pompe (GAA knockout) mice showed that HP-GAA was not as effective due to the fact that it mainly contained high-mannose type glycans, which led to the nonproductive targeting by MRs on endothelial cells and macrophages (21). This suggested that 
GlcNAc-1-phosphotransferase recognizing high-mannose type glycans at the specific sites within the conformational motif $(10,11)$ should be engineered to convert the glycans at all sites to M-6-P glycans in order to avoid MR binding.

In contrast to the unsuccessful targeting of HP-GAA, a series of approaches employing chemical conjugation of M-6-P glycans have been shown to improve the clearance of accumulated glycogen in the skeletal muscles of Pompe mice as well as MPR binding and subsequent targeting to lysosomes (28-32). In a proof-of-concept study, Genzyme researchers isolated M-6-P glycans from recombinant GLA (Agalsidase beta) and, after derivatization to glycosylhdrazines, attached these M-6-P glycan to periodate-oxidized sialic acids of GAA using carbonyl chemistry (28). The resulting modified GAA showed an increased affinity for Cl-MPR and an improved clearance of glycogen in Pompe mice. However, the process of M-6-P glycan isolation from recombinant GAA was not appropriate for scale-up and the isolated glycans were highly heterogeneous. To overcome these limits, instead of natural M-6-P glycans, the researchers conjugated a synthetic M-6-P glycan optimized for the Cl-MPR binding. It contains a hexamannose structure linked to two phosphates $\left(\mathrm{P}_{2}-\mathrm{Man}_{6} \mathrm{GlcNAc}_{2}\right)$ without unnecessary terminal mannose residues (29). The GAA conjugated to the synthetic M-6-P glycan (neo-GAA) has a higher affinity ( $\left.\mathrm{K}_{d} 2-3 \mathrm{nM}\right)$ for the Cl-MPR than the GAA conjugated to natural M-6-P glycans ( $K_{d} 50-100 \mathrm{nM}$ ). Moreover, conjugation of the synthetic M-6-P glycan did not increase the binding affinity for $M R$, suggesting that the internal mannose residues present in the synthetic M-6-P glycan have a very low affinity for $M R$, unlike free terminal mannose residues found in natural M-6-P glycans. It is an important issue because MR binding leads to unproductive uptake by endothelial cells and macrophages. Compared with unmodified GAA, neo-GAA showed approximately a 20-fold more efficient cellular uptake and a comparable reduction in glycogen levels in Pompe mice, with an approximate 8-fold lower dose in the heart and an approximate 4 -fold lower dose in skeletal muscles.

Since the hydrazone bond used for generation of neo-GAA is relatively unstable under physiological conditions, it was replaced with carbonyl-coupled oxime chemistry, which generated a more stable oxime-neo-GAA (Fig. 2A) (30). Due to the fact that the use of oxime chemistry further improved its affinity for Cl-MPR, oxime-neo-GAA reduced the glycogen level in the skeletal muscles of Pompe mice with an approximate 5 -fold greater potency than the unmodified GAA. This study showed that the chemistry used for M-6-P glycan conjugation is important for therapeutic efficacy. Therefore, in the subsequent study, various chemical conjugation strategies were thoroughly compared (31). M-6-P glycan derivatives containing a thiol-reactive group, succinimide, hydrazide, or aminooxy linkers were conjugated to the free cysteines, lysines, or oxidized $\mathrm{N}$-glycans (containing periodate-oxidized sialic acid or enzymatic-oxidized galactose) of GAA. After the evaluation in vitro and in vivo, oxime-neo-GAA derived from the conjugation of aminooxy M-6-P glycans and periodate-oxidized sialic acids was shown to still have the greatest potency in Pompe mice (31). Clinical trials examining the safety and efficacy of this GAA are currently underway (33). Recently, gly-

(A)

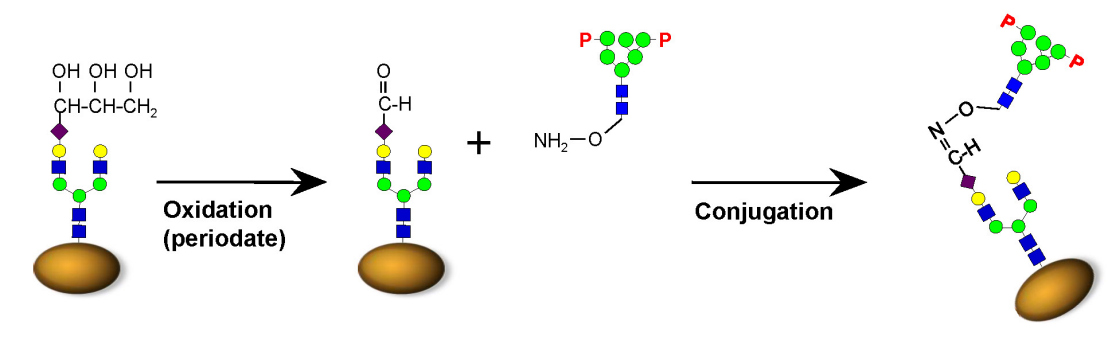

(B)

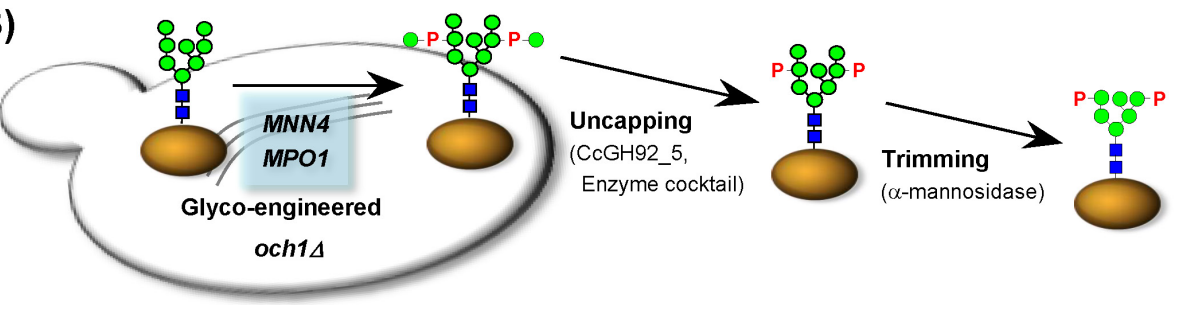

Fig. 2. Schematic representations of glyco-engineering strategies to increase M-6-P glycan content. (A) Terminal sialic acids of GAA were oxidized with periodate to generate the reactive aldehyde group, which reacts with the aminooxy group of the synthetic glycan $\left(\mathrm{P}_{2}-\mathrm{Man}_{6} \mathrm{GlcNAc}_{2}\right)$, generating oxime-neo-GAA. (B) Recombinant enzymes containing mannosylphosphorylated glycans were produced from glyco-engineered yeast. Mannosylphosphorylated glycans of secreted enzymes can be uncapped and trimmed with an uncapping enzyme (such as $\mathrm{CcCH} 92$ 5) and an $\alpha$-mannosidae to generate the M-6-P glycan structure optimized for Cl-MPR binding and cellular uptake. Symbols are identical to those used in Fig. 1. 
can structures for efficient Cl-MPR binding were determined using the chemical conjugation of various glycans containing phosphate groups (32). Zhou et al., reported that the tightest binding to Cl-MPR was achieved with a hexamnnose structure containing two phosphates, while the phosphorylated dimannose moiety appears to be the minimal structure for binding.

Besides the enzymatic engineering and chemical conjugation strategies described above, approaches employing the reconstruction of the yeast glycosylation pathway have been highlighted due to the fact that it generated a high content of M-6-P glycans (34-38). Although yeasts do not have M-6-P glycans, they have high-mannose type glycans containing a mannosylphosphate linked to a mannose residue (mannose-1-phosphate-6-O-mannose) which differs from M-6-P (phosphate-6-Omannose) by the presence of an outer mannose at the non-reducing end. Therefore, through uncapping of this outer mannose, the mannosylphosphorylated mannose structure can be converted to the M-6-P moiety.

In order to generate recombinant yeasts producing enzymes carrying M-6-P glycans, three steps of engineering were carried out $(34,35,37,38)$. Firstly, the genes (such as OCH1 and MNN1) involved in the synthesis of yeast-specific glycan structures were disrupted. Secondly, the gene (such as MNN4, PNO1, or MPO1) enhancing mannosylphosphorylation was overexpressed. Finally, the outer mannose of the mannosylphosphorylated glycans was uncapped in vitro to expose the phosphate group. For this uncapping step, Chiba et al. used an enzyme cocktail secreted from a soil bacterium, which was the first successful engineering of the traditional yeast Saccharomyces cerevisiae to produce a GLA containing M-6-P glycans (34). Recently, Dr. Callewaert's group in Belgium identified a glycosidase (CcGH92 5) with uncapping activity from Cellulosimicrobium cellulans (37). They showed that the recombinant GLA, which was produced from glyco-engineered Yarrowia lipolytica and modified by an in vitro process using the recombinant $\mathrm{CcGH} 92 \_5$ and $\alpha$-mannosidase, has $>80 \%$ of M-6-P glycans (containing at least one M-6-P), corresponding to an approximate 15-fold higher M-6-P content than the approved GAA (Aglucosidase alfa) for Pompe disease. This yeast-generated GAA was delivered much more efficiently to lysosomes of Pompe patient's fibroblasts, and cleared the glycogen accumulated in the heart and muscles of Pompe mice with improved efficacy compared with Aglucosidase alfa (37). This promising result encouraged them to pursue preclinical and clinical development (37).

\section{CONCLUSION}

The successful treatment of LSDs using ERT began with the glyco-engineering of Gcase to contain $\mathrm{N}$-glycans with terminal mannose residues for efficient targeting to macrophages, the most severely affected cells in Gaucher disease. For the other therapeutic enzymes, glyco-engineering strategies to increase
M-6-P glycan content have been actively explored due to the fact that their lysosomal targeting depends on the binding to $\mathrm{Cl}-\mathrm{MPR}$ at the plasma membrane, which is a prerequisite for efficient digestion of lysosomal storages. A chemical conjugation strategy of the synthetic M-6-P glycan optimized for Cl-MPR binding resulted in the development of oxime-neoGAA with an approximate 5-fold greater potency. The strategy employing glyco-engineered yeast also produced GAA with a 15-fold higher M-6-P content, which greatly improved lysosomal delivery and therapeutic efficacy in Pompe mice. Such approaches show promise for the development of next generation therapeutic enzymes with improved efficacy for LSDs.

\section{ACKNOWLEDGEMENTS}

This work was supported by the grants from the Ministry of Trade, Industry \& Energy [10048311] and the Next-Generation BioGreen 21 Program of the Rural Development Administration (PJ011078) in Republic of Korea.

\section{REFERENCES}

1. Ohashi T (2012) Enzyme replacement therapy for lysosomal storage diseases. Pediatr Endocrinol Rev 10 Suppl 1, 26-34

2. Ortolano S, Vieitez I, Navarro C and Spuch C (2014) Treatment of lysosomal storage diseases: recent patents and future strategies. Recent Pat Endocr Metab Immune Drug Discov 8, 9-25

3. Baldo BA (2015) Enzymes approved for human therapy: indications, mechanisms and adverse effects. BioDrugs 29, 31-55

4. Mechler K, Mountford WK, Hoffmann GF and Ries M (2015) Pressure for drug development in lysosomal storage disorders - a quantitative analysis thirty years beyond the US orphan drug act. Orphanet J Rare Dis 10, 46

5. Braulke T and Bonifacino JS (2009) Sorting of lysosomal proteins. Biochim Biophys Acta 1793, 605-614

6. Kim JJ, Olson LJ and Dahms NM (2009) Carbohydrate recognition by the mannose-6-phosphate receptors. Curr Opin Struct Biol 19, 534-542

7. Coutinho MF, Prata MJ and Alves S (2012) Mannose-6phosphate pathway: a review on its role in lysosomal function and dysfunction. Mol Genet Metab 105, 542-550

8. Tiede S, Storch S, Lubke T et al (2005) Mucolipidosis II is caused by mutations in GNPTA encoding the alpha/beta GlcNAc-1-phosphotransferase. Nat Med 11, 1109-1112

9. Kudo M, Bao M, D'Souza A et al (2005) The alpha- and beta-subunits of the human UDP-N-acetylglucosamine: lysosomal enzyme $\mathrm{N}$-acetylglucosamine-1-phosphotransferase [corrected] are encoded by a single cDNA. J Biol Chem 280, 36141-36149

10. Qian Y, Lee I, Lee WS et al (2010) Functions of the alpha, beta, and gamma subunits of UDP-GlcNAc:lysosomal enzyme N-acetylglucosamine-1-phosphotransferase. J Biol Chem 285, 3360-3370

11. Sommerlade HJ, Selmer T, Ingendoh A et al (1994) Glyco- 
sylation and phosphorylation of arylsulfatase A. J Biol Chem 269, 20977-20981

12. Lee $K$, Jin $X$, Zhang $K$ et al (2003) A biochemical and pharmacological comparison of enzyme replacement therapies for the glycolipid storage disorder Fabry disease. Glycobiology 13, 305-313

13. Sakuraba H, Murata-Ohsawa M, Kawashima I et al (2006) Comparison of the effects of agalsidase alfa and agalsidase beta on cultured human Fabry fibroblasts and Fabry mice. J Hum Genet 51, 180-188

14. Sohn Y, Lee JM, Park HR, Jung SC, Park TH and Oh DB (2013) Enhanced sialylation and in vivo efficacy of recombinant human alpha-galactosidase through in vitro glycosylation. BMB Rep 46, 157-162

15. Vedder AC, Linthorst GE, Houge G et al (2007) Treatment of Fabry disease: outcome of a comparative trial with agalsidase alfa or beta at a dose of $0.2 \mathrm{mg} / \mathrm{kg}$. PLoS One 2, e598

16. Togawa T, Takada M, Aizawa $Y$, Tsukimura T, Chiba $Y$ and Sakuraba H (2014) Comparative study on mannose 6-phosphate residue contents of recombinant lysosomal enzymes. Mol Genet Metab 111, 369-373

17. Lauer RM, Mascarinas T, Racela AS, Diehl AM and Brown BI (1968) Administration of a mixture of fungal glucosidases to a patient with type II glycogenosis (Pompe's disease). Pediatrics 42, 672-676

18. de Barsy $T$, Jacquemin $P$, Van Hoof $F$ and Hers HG (1973) Enzyme replacement in Pompe disease: an attempt with purified human acid alpha-glucosidase. Birth Defects Orig Artic Ser 9, 184-190

19. Van den Hout JM, Kamphoven JH, Winkel LP et al (2004) Long-term intravenous treatment of Pompe disease with recombinant human alpha-glucosidase from milk. Pediatrics 113, e448-457

20. Kishnani PS, Nicolino M, Voit T et al (2006) Chinese hamster ovary cell-derived recombinant human acid alpha-glucosidase in infantile-onset Pompe disease. J Pediatr 149, 89-97

21. McVie-Wylie AJ, Lee KL, Qiu H et al (2008) Biochemical and pharmacological characterization of different recombinant acid alpha-glucosidase preparations evaluated for the treatment of Pompe disease. Mol Genet Metab 94, 448-455

22. Tekoah Y, Tzaban S, Kizhner T et al (2013) Glycosylation and functionality of recombinant beta-glucocerebrosidase from various production systems. Biosci Rep 33

23. Grabowski GA, Golembo M and Shaaltiel Y (2014) Taliglucerase alfa: an enzyme replacement therapy using plant cell expression technology. Mol Genet Metab 112, 1-8

24. Berger J, Stirnemann J, Bourgne C et al (2012) The uptake of recombinant glucocerebrosidases by blood monocytes from type 1 Gaucher disease patients is variable. $\mathrm{Br}$ J Haematol 157, 274-277

25. Van Patten SM, Hughes $H$, Huff MR et al (2007) Effect of mannose chain length on targeting of glucocerebrosidase for enzyme replacement therapy of Gaucher disease.
Glycobiology 17, 467-478

26. Koeberl DD, Luo X, Sun B et al (2011) Enhanced efficacy of enzyme replacement therapy in Pompe disease through mannose-6-phosphate receptor expression in skeletal muscle. Mol Genet Metab 103, 107-112

27. Chavez CA, Bohnsack RN, Kudo M, Gotschall RR, Canfield WM and Dahms NM (2007) Domain 5 of the cation-independent mannose 6-phosphate receptor preferentially binds phosphodiesters (mannose 6-phosphate $\mathrm{N}$-acetylglucosamine ester). Biochemistry 46, 12604-12617

28. Zhu Y, Li X, Kyazike J et al (2004) Conjugation of mannose 6-phosphate-containing oligosaccharides to acid alpha-glucosidase improves the clearance of glycogen in pompe mice. J Biol Chem 279, 50336-50341

29. Zhu Y, Li X, McVie-Wylie A et al (2005) Carbohydrate-remodelled acid alpha-glucosidase with higher affinity for the cation-independent mannose 6-phosphate receptor demonstrates improved delivery to muscles of Pompe mice. Biochem J 389, 619-628

30. Zhu Y, Jiang JL, Gumlaw NK et al (2009) Glycoengineered acid alpha-glucosidase with improved efficacy at correcting the metabolic aberrations and motor function deficits in a mouse model of Pompe disease. Mol Ther 17, 954-963

31. Zhou Q, Stefano JE, Harrahy J et al (2011) Strategies for Neoglycan conjugation to human acid alpha-glucosidase. Bioconjug Chem 22, 741-751

32. Zhou Q, Avila LZ, Konowicz PA et al (2013) Glycan structure determinants for cation-independent mannose 6-phosphate receptor binding and cellular uptake of a recombinant protein. Bioconjug Chem 24, 2025-2035

33. Kishnani PS and Beckemeyer AA (2014) New therapeutic approaches for Pompe disease: enzyme replacement therapy and beyond. Pediatr Endocrinol Rev 12 Suppl 1, 114124

34. Chiba Y, Sakuraba H, Kotani M et al (2002) Production in yeast of alpha-galactosidase A, a lysosomal enzyme applicable to enzyme replacement therapy for Fabry disease. Glycobiology 12, 821-828

35. Akeboshi H, Kasahara Y, Tsuji D et al (2009) Production of human beta-hexosaminidase A with highly phosphorylated $\mathrm{N}$-glycans by the overexpression of the Ogataea minuta MNN4 gene. Glycobiology 19, 1002-1009

36. Tsukimura T, Kawashima I, Togawa T et al (2012) Efficient uptake of recombinant alpha-galactosidase A produced with a gene-manipulated yeast by Fabry mice kidneys. Mol Med 18, 76-82

37. Tiels P, Baranova E, Piens K et al (2012) A bacterial glycosidase enables mannose-6-phosphate modification and improved cellular uptake of yeast-produced recombinant human lysosomal enzymes. Nat Biotechnol 30, 1225-1231

38. Gil JY, Park JN, Lee KJ et al (2015) Increased mannosyIphosphorylation of $\mathrm{N}$-glycans by heterologous expression of YIMPO1 in glyco-engineered Saccharomyces cerevisiae for mannose-6-phosphate modification. J Biotechnol 206, $66-74$ 